\title{
Analysis on the Influencing Factors of Acceptance Willingness of Advertisement in WeChat Moments based on Theory of Planned Behavior
}

\author{
Yidi Tang \\ School of Journalism, Communication and Film \& Television, Hainan Normal University, Hainan, \\ China
}

1114837728@qq.com

\begin{abstract}
In the era of the Internet, advertisements in WeChat moments hold been pouring in and favored by businesses. But as the number of advertisements in WeChat moments increases, the quality is uneven. Users have different attitudes towards it, and some users even loathe it. How to meet the needs of users, accurately capture the interests of users and improve the acceptance of users is still an urgent problem to be discussed. Based on the theoretical framework of planned behavior and questionnaire survey, this paper explores the influencing factors of acceptance willingness of advertisement in the information flow of WeChat moments. Research shows that control belief has the most significant impact on users' acceptance willingness. Different age and income levels also have impacts on users' acceptance willingness. Young and middle-aged users and middle-income groups have a higher degree of acceptance of WeChat advertisement. Therefore, opinion leaders can be used to positively guide users to accept the subjective norms of advertisements in WeChat moments. In terms of the content and form of advertisement, researchers can enrich the content of advertisement and simplify the participation steps to improve users' belief in control. In addition, developers can use big data to identify target users and implement precise delivery. Moreover, they can capture users' behavioral willingness to enhance users' acceptance of advertisements in WeChat moments.
\end{abstract}

Keywords: Planned Behavior Theory; WeChat Moments; News Feed Ads; Acceptance Willingness.

\section{Introduction}

WeChat advertisement mainly includes two forms, WeChat moments advertisement and advertisements on the official accounts subscribed. With the rapid development of the Internet, advertisements in WeChat moments appear frequently. Compared with traditional forms of advertisement, it has the advantages of fast dissemination, accurate positioning and strong interactivity, and can collect feedback data through user interaction to improve the return rate of advertisement. This study tries to explore the following questions. First, what are the factors that affect the willingness to accept advertisements in WeChat moments? Second, how do these factors affect user behavior? Third, how to improve users' acceptance of advertisements in WeChat moments? In order to solve the above problems, this paper explores it based on the theoretical framework of planned behavior theory. To some extent, the research perspective of advertisement in moments is enriched. Through questionnaire survey and data analysis, factors affecting user acceptance degree are clarified, and feasible suggestions are provided for improving user acceptance degree of advertisement in WeChat moments.

\section{Literature Review}

The main purpose of this study is to explore the factors that influence the acceptance degree of advertisement users in WeChat moments. Many scholars have used different models and taken different perspectives to study advertisements in WeChat moments. Based on the theory of planned behavior, this paper will analyze and verify the factors affecting user acceptance by combing existing relevant literature and relying on the model called theory of planned behavior (TPB) and using questionnaire survey method to collect relevant data and integrate data. 


\subsection{Planned Behavior Theory and Related Research}

The theory of reasoned action (TRA) was proposed by Professor Ajzen and Professor Fishbein in 1980. In 1985, Professor Ajzen established the theory of planned behavior (TPB) based on the theory of reasoned action with supplementation and modification. The theory of planned behavior includes five related variables: attitude, subjective norms, perceived behavioral control, willingness and behavior. In psychology, attitude refers to the possibility that an individual thinks certain expectations are true [1]. If consumers have a high degree of satisfaction with a product or service, their attitude is positive. On the contrary, it is negative. Subjective norms refer to the social pressure people feel on whether or not to carry out a certain activity. People often care about what the people around them think, and that's what subjective norms are all about. Perceived behavioral control (PBC) is a person's perception of how easy it is to perform a particular behavior, measuring whether it is certain to accomplish something and evaluating it before taking action. When they have a lot of experience opportunities and resources, the obstacles are reduced and the degree of perceptual behavioral control is higher. On the contrary, the degree of perceived behavioral control will be reduced. Attitude, subjective norms, and perceived behavioral control all affect willingness, and willingness directly affects behavior, the target variable. TPB models are designed to study the effects of these or new variables on behavior.

At present, TPB model has been widely used in various subjects. In an advertisement research, Zhang explores consumers' willingness to consume online celebrity beverage advertisements by using research methods such as questionnaire survey and in-depth interview combined with TPB model. Studies have shown that perceived usefulness, perceived trustworthiness and perceived interactivity have positive effects on consumers' behavior. Perceived entertainment does not affect consumers' behavior and attitude. Consumers' behavioral attitudes, subjective norms and perceived behavioral control will positively affect their consumption willingness [2]. In the study of book sales, Niu used reverse thinking and reverse decomposition based on TPB model. He studied that consumers' purchase willingness for books is affected by attitudes (preferences for books, curiosity), subjective norms (guidance by opinion leaders, etc.), and perceptual behavioral control (book prices, purchasing channels, etc.). From this reverse push and from these three aspects to develop relevant strategies. Thus, it will help merchants to promote consumers' purchase willingness for books and finally realize the behavior of buying books [3].

Through literature retrieval, some scholars have used the modified model of TPB theory to conduct relevant research on the acceptance willingness of advertisements in WeChat moments. However, there are few researches on acceptance willingness of advertisements in WeChat moments based on TPB theoretical model. This paper will rely on the existing research framework, through in-depth analysis of the research results and research history of this theory to formulate the research content suitable for the research direction of this paper.

\subsection{Research on WeChat Moments Advertisement}

On January 25, 2015, WeChat Moments Advertisement was officially launched by WeChat as a mobile information stream advertisement. It is a form of advertisement propagated by WeChat advertisement system based on WeChat moments, which is released and managed through WeChat advertisement system [4]. News feed ads, which first appeared on Facebook, are also known as Feed ads. As it is involved in the content that users want to read, its advertisement attributes are easily ignored by users [5]. Moreover, users can interact with the ads. In addition to WeChat moments, news feed ads frequently appear on platforms such as Weibo, Qzone and various APP clients.

With the change of media technology and the popularity of smart devices, the era of WeChat comes. WeChat moments advertisement has the advantages of large number of potential customers, high accuracy of marketing positioning, strong interactivity, effective communication and diversified marketing forms [6]. There are various forms of advertisements in WeChat moments, which are more interactive with users than traditional forms of advertisement communication. If WeChat 
advertisements appear frequently and of low quality in private WeChat moments, users will also have resistance. Therefore, relevant factors affecting users' willingness to accept should be explored.

There are many researched related to WeChat advertisement. Many scholars cut in from different angles, using different models to carry out relevant analysis. Based on social identity cognition and UTAUT model, Lu found that community influence has the greatest influence on advertisement acceptance, and identity perception as an intermediate variable also affects user participation to a certain extent [7]. Wu et al. also used this model to analyze the acceptance willingness of native advertisements in WeChat moments. The study found college students were also less receptive. The main variables affecting their willingness were performance expectation, perceived risk, advertisement reward and social presence. However, effort expectation and community influence have no significant influence on their willingness [8]. In addition to UTAUT model, some scholars also use SWOT model, TAM model, CCSSI model, or TPB model to analyze the user participation degree of advertisements in WeChat moments. In addition to the diversity of research models, there are also diversified research perspectives for the study of advertisements in WeChat moments. For example, the advertisement strategy of WeChat moments, research on the social attributes of WeChat moments advertisement, research on the influencing factors of advertisement sharing behavior and research on the avoidance response of WeChat moments advertisement.

To sum up, many domestic scholars have analyzed advertisements in WeChat moments from different perspectives and models. For example, the UTAUT model is used to study the native advertisements in WeChat moments, the revised model is used to analyze the advertisements in WeChat moments, and the SWOT analysis method is used to analyze the user participation in the news feed ads in WeChat moments. In addition, some scholars have carried out studies on the influencing factors of the sociability of video advertisements in information flow, the influencing factors of advertisement sharing behaviors of users in WeChat moments, and the influencing factors of user engagement. However, explorations on acceptance willingness of advertisements in WeChat moments based on TPB model is relatively lacking. Therefore, it is necessary to combine TPB model with user acceptance willingness of advertisements in WeChat moments.

\section{Research Design}

This study mainly uses questionnaire survey to collect data. The study is mainly based on the theoretical framework of behavioral planning theory (see Figure 1) and combined with reference to relevant literature and practice to set questionnaires. The data were collected and analyzed.

The questionnaire design of this paper is based on the research hypothesis and the above analysis, combined with the recent network related data, referring to the existing literature to carry out multidimensional design of the measurement items. And finally, the questionnaire is formed, consists of three parts.

The first part is a brief explanation of the questionnaire and a visual display to remind interviewees of the general form and content of advertisements in the WeChat moments.

The second part is the core of the data research in this paper, mainly collecting relevant data from three aspects:

(1) Basic using condition of WeChat: This part aims to understand the subjects' use of WeChat, frequency of use, browsing frequency of moments, etc.

(2) Relevant investigation on advertisements in WeChat moments based on the theoretical framework of planned behavior: the influencing factors of users' acceptance willingness were explored by designing four parts: behavior attitude scale, subjective norm scale, perceived behavior control scale and behavior willingness scale.

The third part is the basic information of the interviewees. The questionnaire explores the influence of user gender, age, disposable monthly income and other aspects on the willingness to accept advertisements in WeChat moments. 
A total of 224 questionnaires were collected. Invalid questionnaires were removed based on the principle of "the questionnaire's finishing time is less than $50 \mathrm{~s}$ " and "repeated responses from the same IP address". There were 198 valid questionnaires with effective recovery of $88.4 \%$.

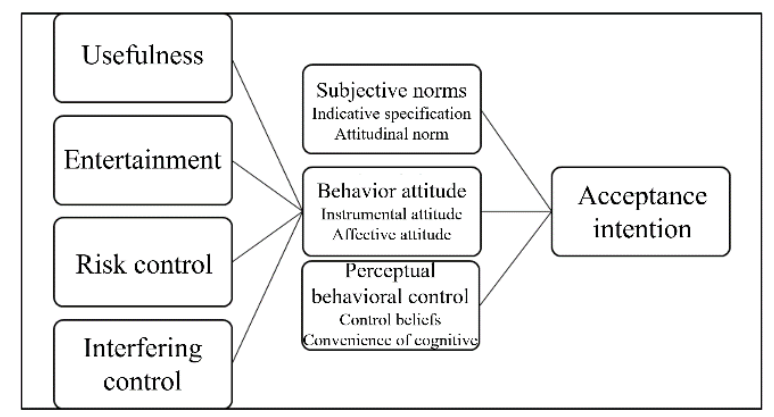

Figure 1. Construction Model of Planned Behavior Theory

\section{Data Analysis}

\subsection{Reliability and Validity Test}

\subsubsection{Reliability Test}

In the questionnaire design of this study, a total of 31 multiple choice questions were set to measure the willingness of respondents to accept advertisements in WeChat moments. Then the reliability was analyzed by using Cronbach's $\alpha$ coefficient. The results show that the overall reliability coefficient of the questionnaire is 0.925 (see Table 1), which is relatively good.

Table 1. Reliability Analysis Table of Questionnaire

\begin{tabular}{|c|c|c|}
\hline & Number of terms & Cronbach's $\alpha$ coefficient \\
\hline Questionnaire overall & 31 & 0.925 \\
\hline
\end{tabular}

\subsubsection{Validity Analysis}

The KMO value of the questionnaire in this paper was 0.919, greater than 0.7, and the Bartlett sphericity test chi-square value was 5114.234. The significance $P$ value was $0.000<0.001$, indicating that the questionnaire had good validity. (See Table 2).

Table 2. Validity Analysis Table of Questionnaire

\begin{tabular}{|c|c|c|c|}
\hline \multirow{2}{*}{ KMO sampling suitability quantity } & \multicolumn{3}{|c|}{ Bartlett sphericity test } \\
\cline { 2 - 4 } & Approximately chi-squared $\chi^{2}$ & .df & Sig. \\
\hline 0.919 & 5114.234 & 45 & 0.000 \\
\hline
\end{tabular}

\subsection{Sample Descriptive Statistics}

\subsubsection{Statistical Analysis of Demographic Characteristics}

In order to analyze whether the sample can meet the diversity requirements, the basic information of the interviewees is statistically analyzed. If the diversity needs cannot be met, targeted questionnaires will be issued. The following data are obtained through certain data collation. The effective questionnaire of this survey basically meets the needs of sample diversity. Specific data analysis is shown in Table 3.

\subsubsection{Analysis of WeChat Usage}

The respondents were all WeChat users. In general, the respondents use WeChat for a long time and browse advertisements in moments more frequently. They are familiar with the WeChat moments. Specific data analysis is shown in Table 4: 
Table 3. Statistical Analysis of Demographic Characteristics

\begin{tabular}{|c|c|c|c|}
\hline Demographics & Category & Frequency & Percentage \\
\hline \multirow{2}{*}{ Gender } & Male & 73 & $36.87 \%$ \\
\hline & Female & 125 & $63.13 \%$ \\
\hline \multirow{5}{*}{ Age } & Under 18 & 2 & $1.01 \%$ \\
\hline & $18-24$ & 132 & $66.67 \%$ \\
\hline & $25-35$ & 39 & $19.7 \%$ \\
\hline & $36-50$ & 21 & $10.61 \%$ \\
\hline & 51 or above & 4 & $2.02 \%$ \\
\hline \multirow{4}{*}{ Record of formal schooling } & High school and below & 22 & $11.11 \%$ \\
\hline & College & 35 & $17.68 \%$ \\
\hline & Undergraduate & 122 & $61.62 \%$ \\
\hline & Postgraduate and above & 19 & $9.6 \%$ \\
\hline \multirow{4}{*}{ Profession } & Student & 131 & $66.16 \%$ \\
\hline & Office worker & 48 & $24.24 \%$ \\
\hline & Freelancer & 10 & $5.05 \%$ \\
\hline & Other & 9 & $4.55 \%$ \\
\hline \multirow{4}{*}{ Monthly disposable income } & $<1500 \mathrm{RMB}$ & 67 & $33.84 \%$ \\
\hline & $1501-3000 \mathrm{RMB}$ & 82 & $41.41 \%$ \\
\hline & $3001-5000 \mathrm{RMB}$ & 30 & $15.15 \%$ \\
\hline & $>5001 \mathrm{RMB}$ & 19 & $9.6 \%$ \\
\hline
\end{tabular}

Table 4. Data Statistics of WeChat Usage

\begin{tabular}{|c|c|c|c|}
\hline Usage status & Category & Frequency & Percentage \\
\hline \multirow{4}{*}{ WeChat usage time } & Within 1 year & 2 & $1.01 \%$ \\
\cline { 2 - 4 } & $1-3$ years & 20 & $10.1 \%$ \\
\cline { 2 - 4 } & $4-5$ years & 42 & $21.21 \%$ \\
\cline { 2 - 4 } & 5 years or more & 134 & $67.68 \%$ \\
\hline \multirow{4}{*}{ WeChat use frequency } & Never & 0 & $0 \%$ \\
\cline { 2 - 4 } & Once in a while & 15 & $7.58 \%$ \\
\cline { 2 - 4 } & Often & 74 & $37.37 \%$ \\
\cline { 2 - 4 } & Always & 109 & $55.05 \%$ \\
\hline \multirow{4}{*}{ WeChat moments browsing frequency } & Never & 0 & $0 \%$ \\
\cline { 2 - 4 } & Once in a while & 43 & $21.72 \%$ \\
\cline { 2 - 4 } & Often & 68 & $34.34 \%$ \\
\cline { 2 - 4 } & Always & 87 & $43.94 \%$ \\
\hline
\end{tabular}

\subsection{Data Analysis}

As a kind of news feed ads, the advertisements of WeChat moments have taken up the trend of blowout growth in WeChat moments. The acceptance willingness of the audience of advertisements in WeChat moments is affected by a variety of factors. According to the data of the questionnaire, the following conclusions can be drawn:

Firstly, the middle-income user group is more receptive to the advertisements in the WeChat moments, and thinks that the advertisements in the WeChat moments can provide certain consumption reference for them. According to the cross analysis of monthly disposable income and behavior attitude scale of WeChat moments advertisement, middle-income users with monthly disposable income of 3001-5000 yuan scored the option of "WeChat moments can make me feel relaxed and happy" (see Figure 2). With an average score of 3.12 points (1-5 points increasing), the item "Advertisements in WeChat moments can be used as my consumption reference" also ranked first with an average score of 3.38. Lower-income users with a monthly disposable income of less than 1,500 yuan scored an average of 2.74 points for this option (see Figure 3 ).

Therefore, different income groups of users have different consumption patterns and consumption concepts. All middle-income users have the highest willingness to accept advertisements in WeChat moments, believing that the news feed ads in WeChat moments can provide them with some 
consumption references and help them choose their favorite products. Higher income groups do not reject the WeChat moments advertisement. However, the lower income consumer group has a low willingness to accept the advertisements in the WeChat moments, has a certain resistance to the news feed ads in the WeChat moments, and does not like to be induced by the advertisement.

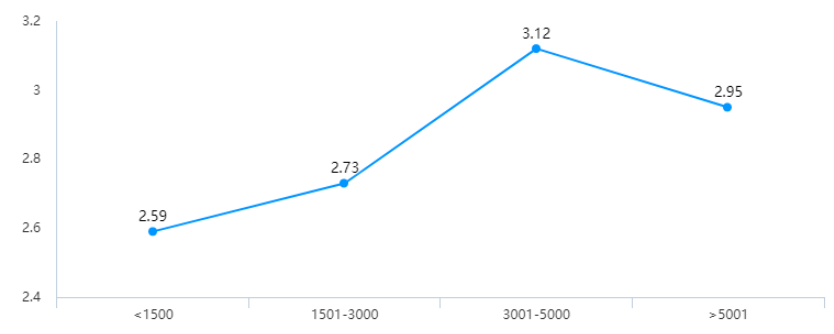

Figure 2. Analysis of disposable income on affective attitude to advertisements in moments in months

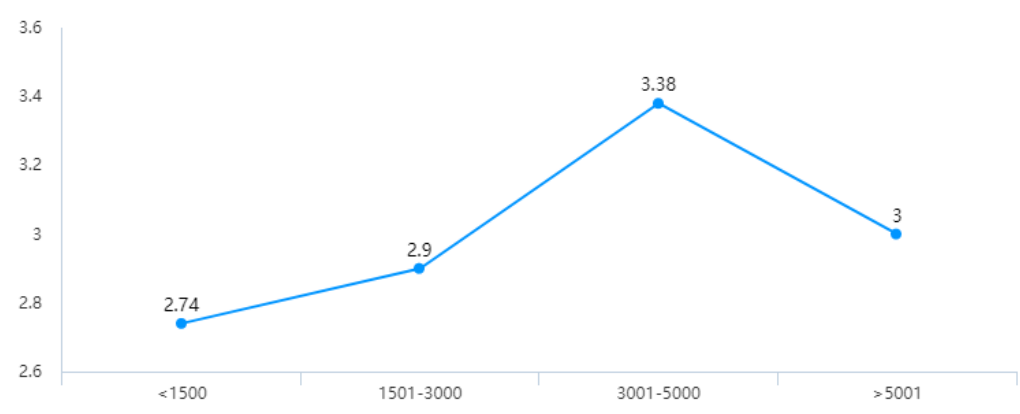

Figure 3. Analysis of disposable income on instrumental attitude to advertisements in moments in months

Secondly, control belief has the most significant impact on users' willingness to accept. Statistical analysis of data (see Figure 4) shows that the average score of the matrix of the perceived behavior control scale reaches 3.44 points, which has a greater impact on the acceptance of advertisements in WeChat moments compared with other variables. In the measurement dimension of cognitive convenience, the results show that sufficient time and enough Internet speed and traffic provide cognitive convenience for browsing advertisements in WeChat moments, and cognitive convenience also positively affects the willingness of the audience. In the measurement dimension of controlling belief, the average score of the item "whether to browse, click, comment or share the advertisements in WeChat moments is totally determined by my will" is as high as $3.61,33.48 \%$ of people choose 5 , agreeing with this statement, believing that browsing, clicking and sharing the advertisements in WeChat moments is completely determined by my will. "Can view, like, comment or share WeChat ads according to their own needs and interests" scored 3,41 on average.

Therefore, most users prefer more controllable forms of advertisement, which can be freely selected according to their interests and desires. If the advertisements in WeChat moments is too complicated, it will give users a strong sense of compulsion. In addition, the steps are complex and the operation is difficult, users will reject such advertisements in WeChat moments.

Third, young and middle-aged users have the highest acceptance of WeChat advertisement. According to the statistical analysis of data (see Figure 5 and 6) and the cross analysis of users' age and behavioral willingness to accept advertisements in WeChat moments, young and middle-aged users aged 36-50 scored an average of 3.57 points for the option of "willing to accept advertisements in WeChat moments", ranking first. Users aged 25-35 ranked second, with a higher willingness to accept advertisements in WeChat moments. In addition, compared with the youth group aged 18-24, these users are also more willing to comment and share friends' WeChat moments ads than other user groups. The average score of young users aged 18-24 for "willing to accept advertisements in WeChat moments" and "willing to participate in the interaction of advertisements in WeChat moments" is 
only 2.66, and the willingness to accept and interact with advertisements in WeChat moments is the lowest.

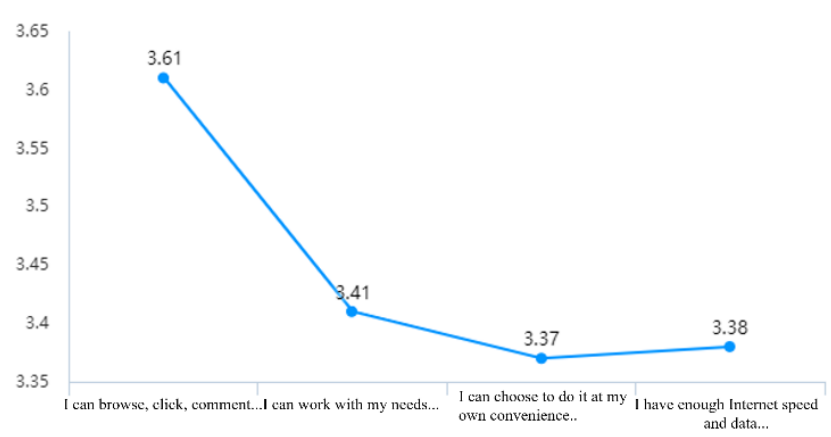

Figure 4. Perceptual Behavior Control Scale Analysis of Influencing Factors oof Acceptance Willingness of Advertisements in Moments

Different age groups have different consumption concepts, and young and middle-aged groups are more likely to be attracted by advertisements. For example, if some ads are placed with offers, such users will be interested in them, believing that news stream ads can help them choose a relatively good deal. However, young users have more diversified channels for product understanding and have more ways to refer to products. In addition, the products promoted by advertisements are not necessarily what users need, and young consumer groups are more inclined to buy the products they like. As a result, news feed ads do not attract younger users and its acceptance is not high. Therefore, the advertisements should be accurately positioned and delivered according to the target audience to meet the needs of users of different ages.

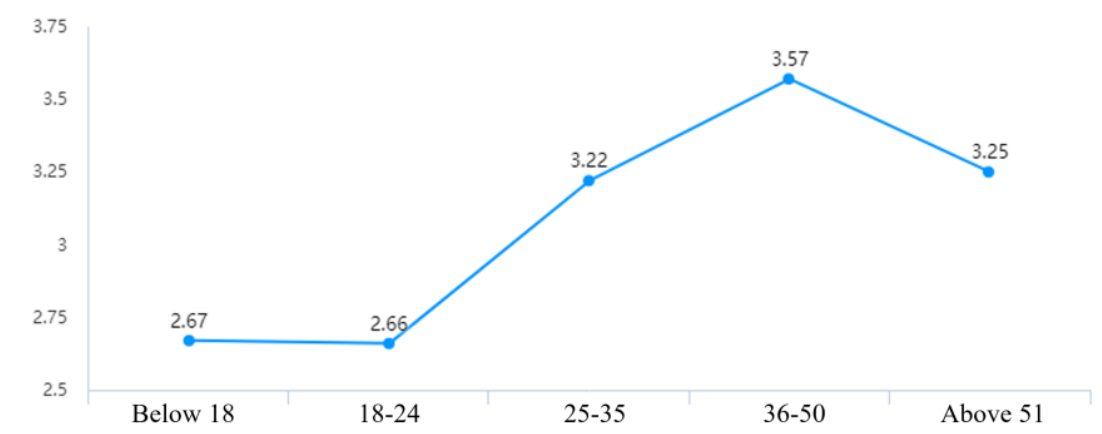

Figure 5. Statistical Analysis of Acceptance Willingness of Different Age Groups to Advertisements in WeChat Moments

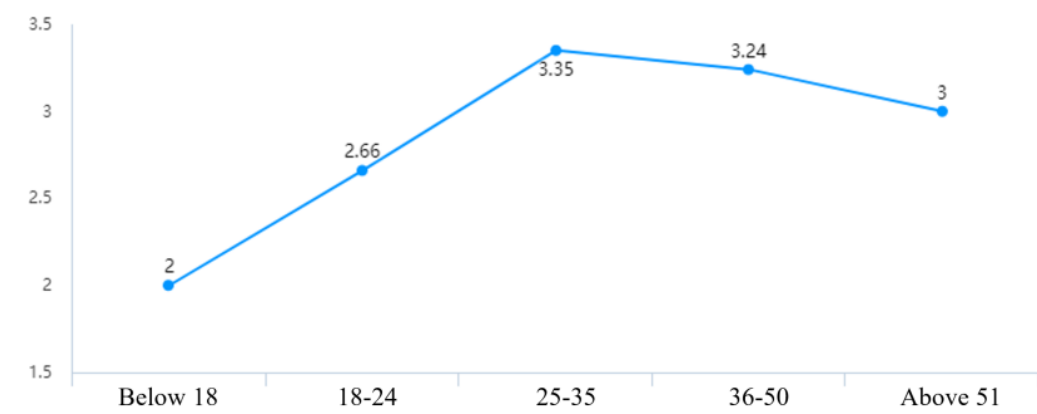

Figure 6. Statistical Analysis of Interaction Willingness of Different Age Groups for Advertisements in WeChat Moments 


\section{Implication and Discussion}

According to the above data analysis and relevant conclusions, based on the research on acceptance willingness of advertisements in WeChat moments based on the theoretical framework of planned behavior, control belief has the most significant impact on users' acceptance intention, and young and middle-aged groups and middle-income groups have higher acceptance willingness to advertisements in WeChat moments. In order to improve the acceptance willingness of users, we can start from the guidance of opinion leaders, the content and form of advertisements, and the precise delivery of advertisements.

\subsection{Opinion Leaders' Positively Guiding Users to Accept the Subjective Norms of Advertisements in Moments}

The concept of opinion leader was first proposed in People's Choice. In communication, it is defined as a person who is active in interpersonal communication network, often provides information, views or suggestions to others and exerts personal influence on others. [9] At first, the application scope of opinion leaders was mainly limited to the political field, and later expanded to other fields such as shopping. According to the data survey, subjective normative behavior has a positive impact on people's willingness to accept advertisements. Therefore, the guidance of opinion leaders can attract more users to participate in and improve the conversion rate of advertisements in WeChat moments.

According to the results of the questionnaire survey, users' attitudes towards advertisements in WeChat moments are largely influenced by people around them. Therefore, opinion leaders influence people's subjective norms during the dissemination of advertisements in WeChat moments, and play an indicative role in the selection of goods. When people have no actual contact with a product, users will have resistance to advertisements in WeChat moments. In this case, the communication of opinion leaders can alleviate people's resistance to products to some extent, narrow the psychological distance between advertisement products and the audience, and enable the audience to gradually contact, understand and accept, and finally reach the purchase behavior. In addition, data show that consumers' belief in the opinion leaders of acquaintances is 18 times higher than that of general advertisement, while their belief in the propaganda of hard advertisements only accounts for 5\% [10]. Compared with celebrity endorsements, consumers and opinion leaders share more common significance in the introduction and promotion of friends and relatives. In the advertisement promotion of WeChat moments, the acquaintance network is more likely to draw the relationship between the audience and the advertisement. Therefore, in the advertisement setting of WeChat moments, the comments or usage suggestions of the opinion leaders of friends can be preferentially displayed. Users feel the pressure from the reference group, which positively affects the subjective norms of consumers and is more likely to stimulate the shopping desire of consumers.

\subsection{Enrich Advertisement Content, Simplify Participation Steps and Improve Users' Belief in Control}

Perceptual behavioral control variables have the most significant influence on the acceptance degree of advertisements in WeChat moments. From the analysis of its control belief dimension, it can be seen that users' participation in advertisements in WeChat moments depends on their own will. From the perspective of cognitive convenience, it can be seen that users will browse advertisements in WeChat moments in their leisure time and when cognitive tools can provide convenience. Therefore, advertisements in WeChat moments should try to cater to users' interest points and improve their willingness to participate. In Ogilvy on Advertising, Ogilvy proposed that the content of an advertisement is more important than the way of presenting the content [12]. More advertisements in the WeChat moments are entering the user's field of vision, especially in the more private areas. So, to let users accept and reach the purchase intention, the content of the advertisements is particularly important. The first thing to catch the attention of the audience is to 
have a distinct style and prominent label, which makes it stand out from homogeneous issues [11]. In terms of content design, the display form of advertisements content can also get rid of the relatively single graphic presentation, and various forms such as small programs, H5 and short videos can be used to enhance the significance of labels and improve the controllability of content interaction [12]. According to the survey results show that the user's control belief affects the acceptance of advertisements WeChat moments, the audience's interest will can lead users browse the thumb up WeChat moments of advertisement, so in the design of advertisements in the form of a WeChat moments, try to enhance its interactive, let users have more engaged, make the user feel interesting. But the steps cannot be too complicated, so as to avoid consumption spending of their patience, which will make it unable to achieve the final purchase behavior.

\subsection{Make Use of Big Data to Identify Target Users and Implement Precise Delivery to Capture User Behavior Intentions}

Compared with traditional forms of advertisement, the current advertisement industry pays more attention to data thinking. Through the Internet, the Internet of things, big data to clarify target users. The behavioral willingness of users is captured, and precise delivery is implemented to achieve higher advertisement returns. Big data refers to "how to capture, manage, process and sort out the information to help the information acquirer or decision maker to achieve a new understanding and understanding of goals in a reasonable time and space after data processing and analysis "[13]. From the perspective of audience's behavioral intention, big data can collect massive and diverse data as a reference, and search and analyze target information. This provides the possibility for the precise placement of advertisement industry. This makes it pay more attention to the differences and interest points of the audience, improve the enthusiasm of the audience to participate in the interaction of advertisements and the willingness to share advertisement.

Control belief has a positive impact on users' behavioral choices, and users are more willing to accept advertisements that meet their psychological expectations. Therefore, WeChat moments can also use the special attributes of big data to launch advertisements, improve users' willingness to participate, and thus better realize the transformation of sales and click rate. Big data can be used to collect multi-platform information, comprehensive data capture of users' browsing information, address positioning, consumption records, demographic characteristics and attributes, and analyze whether this consumer is the target user for advertisement, and can mine potential target users. WeChat moments advertisements can also be placed according to the specific situation of consumers. For example, in the shopping mall, ads owners can choose the advertisements of the corresponding product. In the restaurant, ads owners can choose the corresponding catering advertisement. This can make the WeChat moments advertisements more in line with the psychological needs of consumers, so as to enhance the acceptance of users for the WeChat moments advertisement. Thus, the in-session approach makes users willing to browse and participate in advertisement interaction to generate the final consumption behavior.

\section{Research Limitations}

This paper mainly carried out the research through questionnaire survey. Due to the lack of research ability, this paper still needs to be improved.

First, the sample size of this study is small, which cannot well reflect the actual situation of most users. A total of 224 points were collected and 198 valid questionnaires were collected. In addition, the questionnaire mainly adopts the method of snowball survey, and the small sample size cannot accurately answer the question. Second, there are limitations in sample selection. Due to the limitations of the author, this sample mainly focuses on the youth group aged 18-24, which tends to be young as a whole. Moreover, vocational education has been simplified. However, this kind of crowd cannot represent the general WeChat moments advertisements of the entire audience. Therefore, respondents should cover more different age levels, job types and educational levels to 
improve sample representativeness. Thirdly, in terms of research methods, this paper mainly adopts the questionnaire survey method. Due to the uncertainty of filling the questionnaire and the quality of filling the questionnaire, it is difficult to make a strict judgment. Future research can be combined with in-depth interviews and other methods to further study this problem. Finally, the data analysis is not deep enough in data capture and analysis. The data should not be limited to single analysis, but more in-depth mining and cross-analysis in many aspects.

\section{References}

[1] Yan, Y. A Review on the Origins and Development of the Theory of Planned Behavior. Chinese Journal of Journalism \& Communication, 2014, 36(07): 113-129.

[2] Zhang, B. A study on consumers' acceptance willingness of online black tea beverage brand advertisement from the perspective of planned Behavior Theory. Minzu University of China, 2019.

[3] Niu, X. Research on Book Marketing Communication Strategy Based on Planned Behavior Theory. Publishing Research, 2019(08): 38-43.

[4] Xu, D. A Study on the Influencing Factors of Wechat Users' Willingness to Accept Advertisements in WeChat Moments. Jilin University, 2016.

[5] Kong, Q. Research on News Feed Ads Communication in WeChat Moments. Dalian University of Technology, 2016.

[6] Wang, G. Analysis of communication effect of WeChat advertisement. News Research, 2015(08): 55-56.

[7] Lu, X. Research on User Participation Effect of WeChat Moments Advertisement. Wuhan University, 2018.

[8] Wu, W., Zhou, T. An empirical study on native advertisement acceptance in WeChat moments based on UTAUT model. Advertisement Overview (Theoretical Edition), 2016(05): 41-49.

[9] Guo, Q. Journalism \& Communication (2nd Edition). Beijing: China Renmin University Press, 2011: 189.

[10] Tang, X. On the Function and Application of Personal Communication in Advertisement Communication. Journal of Xiangtan University: Philosophy and Social Sciences, 2005(02): 67-71.

[11] Zhang, M. Advertisement Content and Form co-create Successful Communication: Rethinking David Ogilvy's view of "Content and Form". New Media Research, 2019, 5(15): 104-105.

[12] Feng, L., \& Wu, Q. On Content-based Communication Strategies of WeChat H5 from the Perspective of Audience Psychology. Journal of Hengyang Normal University, 2019, 40(01): 149-152.

[13] Ni, N. Big Data Era and Communication Conceptual Changes. Journal of Northwestern University (Philosophy and Social Sciences), 2014, 44(01): 139-145. 